\title{
Measuring electrochemical noise of a single working electrode for assessing corrosion resistance of polymer coated metals
}

\author{
Sina S. Jamalia ${ }^{a 1}$ Douglas J. Mills ${ }^{a *}$, John M. Sykes ${ }^{b}$
}

\author{
a School of Science and Technology, University of Northampton, St George's Avenue, Northampton, \\ NN2 6JD, UK \\ ${ }^{\mathrm{b}}$ Department of Materials, University of Oxford, Parks Rd, Oxford, OX1 3PH, UK \\ *Corresponding Author: Sina S. Jamali, Tel: +61 426736 630, Fax: +61 24221 3114, Email: \\ sina.jamali@northampton.ac.uk
}

\begin{abstract}
Electrochemical Noise Measurement (ENM) of the spontaneous perturbation of current and potential of coated samples immersed in electrolyte determines the resistance of the coating system. ENM offers several advantages: the measurement is relatively simple to make, it is completely non-interfering with the natural process occurring on the surface and the data are simple to interpret. The original standard arrangement for ENM using a pair of samples has limitations for practical applications because two separate and nominally identical working electrodes are needed and this requirement is very hard (if not impossible) to fulfill in on-site application. This paper describes an alternative approach for electrochemical noise measurement to measure the noise resistance $\left(R_{n}\right)$ of protective coatings based on use of just one working electrode. In this so-called "Single Cell" (SC) arrangement the electrochemical noise current and electrochemical noise potential between the working electrode and a non-noisy reference electrode is measured separately and consecutively. This new approach has been tested for a range of coating resistances. Also, the coating's resistance has been measured using DC resistance and EIS (at low frequency) and the results were compared with the $\mathrm{R}_{\mathrm{n}}$ obtained from the single cell (SC) set up.
\end{abstract}

Keywords: electrochemical noise measurement; single working electrode; data analysis; organic coatings; reference electrode; reproducibility.

\section{Introduction}

Electrochemical noise measurement comprises simultaneous measurement of potential and current fluctuations caused by spontaneous electrochemical reactions. It is accepted as a nondestructive/non-intrusive technique capable of monitoring basic changes in an electrochemically active system. The technique has been used to calculate $R_{n}$ as a measure of corrosion resistance $[1,2]$ and also statistical methods have been applied to evaluate the corrosion regime [3-5].

Since first introduced to the field of protective coatings by Skerry and Eden in 1986 [6] the electrochemical noise method (ENM) has found increasing use as an effective way of assessing the

\footnotetext{
${ }^{1}$ Current address: Sina S. Jamali, Intelligent Polymer Research Institute, AlIM Faculty, Innovation Campus, University of Wollongong, Wollongong, NSW 2522, Australia, Tel: +61 426736630
} 
protection afforded by organic coatings on metals $[7,8]$. It has been shown that the noise resistance conforms with the corrosion resistance from other well-established techniques e.g. EIS $[9,10]$. The usefulness and simplicity of the ENM technique plus the relatively quick measurement and inexpensive instrumentation makes the method potentially ideal for in-situ corrosion assessments. It also offers the advantage of non interfering measurement compared to $D C$ techniques where the applied current/potential alters the system from the steady state. DC measurements may not ideally represent system characteristics in a self-corroding/uninterrupted condition and also more time will be required to reach the steady state condition [10]. ENM also provides mechanistic information about the corroding bare metals (e.g. uniformity/localization of corrosion or at a scribe in a coating) which may not be acquired by the techniques such as EIS and DC measurements. Examples of the recent breakthroughs in this field are novel methods of noise data analysis for studying localized corrosion [11], detecting the initiation of stress corrosion cracking [12] and studying nucleation of pitting corrosion [13].

The ENM technique with the original arrangement, e.g. salt bridge set-up (figure 1), is well established and is commonly applied for studying electrochemical behavior of corroding systems in the laboratory. This method uses two nominally identical but separate working electrodes and works effectively for measurements on bare and coated metals. The current between the two working electrodes is measured by a zero resistance ammeter (ZRA) simultaneously with the potential of these electrodes (now in effect coupled together by the potentiostat) with respect to a noiseless standard electrode (normally a Saturated Calomel Electrode (SCE)). However, it will usually be impractical to find two nominally identical but separate working electrodes in the real on-site applications, e.g. a bridge or a ship hull. The first step towards making the technique more practically useable for organically coated metal on-site was taken by Mills and Mabbutt in 1998 [14]. The socalled "Single Substrate" (S.S.) (Figure 2) arrangement is a re-arrangement of the original salt bridge arrangement. It replaces the working electrodes (the two substrates) by SCEs and uses the substrate as the pseudo reference. Nominally noiseless SCE's make electrolytic contact with the corroding surface and the current perturbation measured by ZRA originates from the electrochemical activity of the two coupled areas of the specimen. This arrangement was also successfully utilized for online monitoring of corrosion behaviour and degradation rate of coated substrates in long term cyclic Prohesion exposure using embedded Pt electrodes by Bierwagen et al. [15]. There has been further work carried out on the validation of the technique, mainly by Mabbutt [16-18].

\section{Figure 1}

Figure 2

Further development of ENM for application to coated metal has been made by Woodcock et al. in 2004 mainly to eliminate the need for electrical connection to the substrate and so make the technique more practically useable [19]. In this new, so-called "NO Connection to Substrate" (NOCS) arrangement, electrochemical noise potential is measured against a third SCE which, similar to the working electrodes, is in electrolytic contact with the specimen. The main advantages of this arrangement are elimination of wired electrical contact to the substrate and, compared with the Single Substrate arrangement and use of a highly stable reference electrode as part of the current measuring circuit. NOCS arrangement for electrochemical noise measurement is shown in Figure 3. 
Figure 3

An overview of the newly developed ENM arrangements and practical approach to implement these for in-situ measurement has been discussed by Mills in Ref. [20]. Despite all the efforts to make ENM a practical on-site method, field evaluation offers a significant challenge, particularly in the case of submerged structures and awkwardly shaped substrates, where providing and isolating the two or three working electrodes (respectively for SS and NOCS arrangements) may be impractical. Also in contrast with most laboratory experiments, field measurements are usually performed on random surfaces with unknown resistance values. So, practically all the proposed noise data collection arrangements introduce a significant uncertainty in regards to which area has dominated the measured $R_{n}$. There have been recent studies to elucidate the dominant factor(s) [21,22] but further investigations are still required to clarify the ambiguity.

In the present work a new arrangement for ENM has been introduced that makes the measurement on a single working electrode. The so-called "Single Cell" (SC) arrangement provides the capability of measuring noise resistance for immersed objects or inside storage tanks without the need for two isolated working electrodes. Also since only one area (WE) is involved in the measurement, it eliminates the uncertainty introduced by multiple working electrodes.

\section{The Single Cell arrangement and data acquisition}

The SC set-up comprises one working electrode and a noiseless reference electrode as shown in figure 4. Unlike previous experimental procedures, the electrochemical noise current (ENC) and electrochemical noise potential (ENP) are not (and cannot be) measured simultaneously, so it is essential to be sure that the corroding sample remains in an unchanged condition throughout the time of experiment. Since the noise data collection is relatively quick (e.g. 512 data point at $2 \mathrm{~Hz}$ takes about $4 \mathrm{~min}$ ) and the process of data collection imposes no external stimulus steadiness of working electrode is a reasonable assumption. In most cases under field conditions the coated structure has been well equilibrated by the time the measurements are made.

The ENP was measured by recording the sample potential against a SCE. This was performed (similar to a conventional three-electrode ENM) under open-circuit conditions; as the SCE generates little noise (ref) only the electrochemical noise generated by the sample is measured. ENC is usually measured with the sample connected to an identical sample via a ZRA, but here ENC cannot be measured directly using a ZRA due to the potential difference between the substrate and the SCE that would generate a DC current flow. Instead, the substrate potential was determined as the mean value of electrochemical noise potential data set, then this was then implemented electronically by the potentiostat acting as a constant potential source, with the SCE serving as both reference and auxiliary electrodes. This is permissible for high resistance coatings where the current is too small to perturb the reference electrode, but a separate auxiliary electrode can easily be added where necessary to give true potentiostatic control. The ENC measurement was carried out by taking 512 current measurements at $2 \mathrm{~Hz}$ with the sample held at the free corrosion potential. Random fluctuations in sample behavior now generate current noise limited by the electrode impedance, instead of potential drift, because the sample is forced to remain at a fixed potential. 
Figure 4

The noise data were treated using the analytical programme "ENANALIZ", developed by Prof. R. Cottis at the School of Materials, University of Manchester. The programme was used to remove DC drift from the noise data in the time domain. The noise resistance was calculated in accordance with Ohm's law, eq. (1).

$$
R_{n}=\frac{\sigma(V)}{\sigma(I)}
$$

where $\sigma(\mathrm{V})$ and $\sigma(\mathrm{I})$ are the standard deviations of potential and current fluctuations.

Also the time domain data was transferred to frequency domain using the fast Fourier transform (FFT) and Maximum Entropy Method (MEM) functions of the programme. The Power Spectral Densities (PSD's) were then generated to calculate the spectral noise resistance, $R_{s n}$, according to eq. (2).

$$
R_{s n}(f)=\sqrt{\frac{\psi_{V}(f)}{\psi_{I}(f)}}
$$

where $\psi_{V}$ and $\psi_{l}$ are the PSD's of potential and noise current respectively. Spectral noise resistance was plotted as a function of frequency and compared to the modulus of impedance $|Z|$ given by EIS. The minimum and maximum frequencies that can be resolved in the frequency domain are given by eq. (3):

$$
f_{\text {min }}=\frac{1}{N \cdot \Delta t}, \quad f_{\text {max }}=\frac{1}{2 \cdot \Delta t}
$$

with $\mathrm{N}$ the total number of samples and $\Delta \mathrm{t}$ the time increment between samples. The spectral noise at the minimum frequency was taken as the DC limit and was compared to the coating resistance given by the $D C$ technique.

Note that the concept of consecutive measurement of electrochemical noise potential and electrochemical noise current may also be applied to a two working electrode arrangement without electrical connection to substrate. Similar to NOCS arrangement (fig. 3), ENP may be measured between two SCEs in electrolytic contact with areas of interest. Subsequently, noise current can be measured between the same two electrodes. One advantage of this (in addition to having no electrical connection to the substrate) could be the less ambiguous result due to using fewer numbers of cells. It should be noted that the previous ENM arrangements have all used more than one working electrode which leaves an uncertainty about which electrode has dominated the result.

\section{Experimental}


A range of coating systems from low to very high protection properties were selected to achieve relatively low, medium, high and very high resistance. The low resistance coatings were a maintenance alkyd paint (low R1) and an alkyd varnish (low R2) based on short oil soya based alkyd resin (supplied by Pronto Paint Co.) on steel Q panel with dry film thickness of about $90 \mu \mathrm{m}$. A single coat epoxy and a two coat epoxy primer/polyurethane topcoat system (both supplied on $Q$ panel by Liquid Plastics Ltd) at overall thicknesses of $290 \mu \mathrm{m}$ and $320 \mu \mathrm{m}$ respectively, were chosen to represent medium and high resistances. The very high resistance coating was a double coat system composed of a glass-flake pigmented polyurea topcoat on an iron oxide primer with overall thickness of about $380 \mu \mathrm{m}$ on a garnet blasted steel panel supplied by Akzo-Nobel. These five coating systems were each used as representive of typical coatings with low, medium, high and very high resistances as measured using a high impedance DC instrument (solid state Keithley Electrometer model $610 \mathrm{C}$ ). They were all immersed in $0.5 \mathrm{M} \mathrm{NaCl}$ solution at least for $48 \mathrm{hr}$ prior to measurement. The exposed area of coating in contact with salt solution was $11.4 \mathrm{~cm}^{2}$.

ENM and EIS measurements were performed using an ACM GillAC electrochemical workstation with a SCE as the reference electrode. Electrochemical noise data were collected at $2 \mathrm{~Hz}$ sampling rate for 512 data points per experiment. The instrumental noise was checked by measuring the noise level without the samples connected. A standard 3 electrode arrangement was used for EIS measurements using a platinum electrode as auxiliary electrode with perturbation amplitude of \pm 10 $\mathrm{mV}$ around OCP within $10 \mathrm{kHz}-10 \mathrm{mHz}$ range of frequency. All the results presented have been corrected for area (i.e. per $\mathrm{cm}^{2}$ ).

\section{Results and discussion}

\subsection{Accuracy of $R_{n}$ measured by SC arrangement}

The accuracy and validity of the method was examined by comparing the $R_{n}$ obtained by SC arrangement with the $R_{n}$ obtained by the Single Substrate arrangement, AC impedance at $0.01 \mathrm{~Hz}$ and the $D C$ resistance. Figure 5 reveals a very good agreement between $R_{n(S C)},|Z|_{0.01 \mathrm{~Hz}}$ and $R_{D C}$ values across the range of coating resistances (coded as low $R$ to very high $R$ ) and it is clear that for the purpose of monitoring coating performance this new method agrees with the others within normal tolerances. Also $R_{n(S S)}$ and $|Z|_{0.01 \mathrm{~Hz}}$ show acceptable conformance with the $R_{n(S C)}$ in most cases, however in some cases $R_{n(S c)}$ does not fit the $R_{n(s S)}$ and $|Z|_{0.01 \mathrm{~Hz}}$ appropriately. It should be noted that some differences could be originated from difference in the means used for measurements. The external potential applied to the sample in DC measurement and sinusoidal potential perturbation applied in EIS measurement may alter the mechanism of ionic conduction inside the polymer film thus resulting in somewhat different resistance values. Also it must be noted that the SC arrangement, AC impedance and the DC measurement only engage one electrochemical cell while the ENM with Single Substrate configuration engages two electrochemical cells. Thereby the $R_{n(S S)}$ can be dominated by either of the values. Observations have shown that the $R_{n(S S)}$ is usually dominated by the cell with the higher resistance value.

$|\mathrm{Z}|_{0.01 \mathrm{~Hz}}$ presents a reasonable agreement with $\mathrm{DC}$ values at low resistances. However, AC impedance shows a high level of uncertainty for high resistance samples. This is perhaps due to the frequency dependence of $|\mathrm{Z}|$ for highly protective coatings with capacitive behaviour. It has been suggested that $R_{n}$ is equal to $R_{s n}$ and the $D C$ limit of the impedance spectrum in the Bode plot only 
when the diffusion process dominates the corrosion process (resistive behaviour) [23-25]. In order to reach the $D C$ limit frequency for such coatings there might be the need for continuing the experiment to lower frequencies, e.g. $0.001 \mathrm{~Hz}$. At such frequencies an EIS measurement will require a very long time to complete and a more sophisticated (and expensive) instrument to be used. Also $\mathrm{AC}$ scanning at very low frequencies is highly sensitive to extraneous noise and other interference.

\section{Figure 5}

\section{2 reproducibility of $\mathrm{SC}$ on similar coatings}

Assessing the performance of a paint coating in practice involves testing different areas of the paint to obtain an average value of protection properties. Therefore, it is crucial for an electrochemical method to reliably and reproducibly examine different areas of the paint with slightly different properties and obtain results within an acceptable tolerance. In the experiments conducted here the SC arrangement was used to examine three different areas of an alkyd paint with $80 \pm 5 \mu \mathrm{m}$ thickness on steel. ENP and ENC were independently measured using SCE at $2 \mathrm{~Hz}$ sampling rate to collect 2048 data sets. DC resistance and AC impedance were also used as complementary tests of accuracy. Figure 6 compares the resistance values acquired by the three techniques for each single cell. In addition to $R_{n(S C)}, R_{n(D C)}$ and $|Z|_{0.01 \mathrm{~Hz}}$, cells were used in 2 and 3 electrodes combinations with single substrate (SS) and NOCS arrangements to provide a comparison between different arrangements of ENM.

The three methods of measuring individual resistance (SC, DC and EIS) show close values (i.e. $R_{n(S C)}$, $R_{n(D C)}$ and $|Z|_{0.01 \mathrm{~Hz}}$, respectively), however they do not rank the coatings similar to another. This could be due to the experimental error or the different nature of each test. For instance DC method only measures the resistance of coating against ionic transport provoked by an external DC potential while ENM also considers the passivation state and stability of potential underneath the paint. Consequently under-film passivation, e.g. at the cathodic sites, may have a significant influence on the resistance measured by ENM while it may be ignored by the DC electrometer. In the case of other ENM arrangements, involvements of more than one working electrode (two electrodes in SS arrangement and three electrodes in NOCS) have resulted in non-identical results when compared to SC arrangement. Table 1 shows the statistical analysis of results obtained by three measurements using each method. Coefficient of variation ( $\mathrm{CoV}$ ) is calculated by dividing the standard deviation by mean value as a normalized measure of dispersion. By definition distributions with CoV $<1$ are considered low-variance, while those with $\mathrm{CoV}>1$ are considered high-variance. All three methods of SC, DC and EIS show low variance in their ability to examine similar paint coatings.

\section{Figure 6}

Table 1

Figures 7a-c provide further detail on the conformance of the EIS and $R_{s n}$ in the frequency domain as well as DC method and $R_{n}$. Reasonable coherence between EIS and $R_{s n}$, within the overlapped area, indicates the promising potential of ENM by SC arrangement as a frequency sensitive method for practical assessments of a single area of interest in the field. 


\subsection{Frequency domain data analysis}

A 2048 point data set acquired from independent ENC and ENP measurements on the epoxy/PU coating system (high R) were transferred into the frequency domain using both FFT and MEM methods and PSD of noise resistance $\left(R_{s n}(f)\right)$ was produced. The Bode plot from the AC impedance experiments is superimposed on the same graph to compare the $R_{s n}(f)$ to the $|Z(f)|$ plot obtained from EIS and check how accurately the Bode plot can be reproduced mathematically from a single cell noise measurement. Although the value from the noise method falls off at the lowest range of frequencies, Figure 8 reveals a reasonable agreement between $R_{s n}(f)$ and $|Z(f)|$ plots. The important implication of this observation is that it indicates the possibility of using noise measurement technique to monitor corrosion behaviour of a single electrode and get the same estimate as one would get using the more complicated and time-consuming $A C$ impedance technique. The similar slopes of PSD plots of current and potential in the low to mid-frequency range (shown in Figure 9) indicate that $R_{n}$ is a frequency independent measure of the system characteristics $[25,26]$.

\section{Figure 8}

\section{Figure 9}

\subsection{Noise data repeatability/reliability}

Since the ENP and ENC data collections are not simultaneous it is crucial to ensure that the corroding system has reached an acceptable steady state. To assess this the set of the 2048 noise data points, used for the frequency domain data analysis, was divided into 8 separate groups of 256 data "sets" and the $R_{n}$ was calculated for each set individually. Figure 10 compares the $R_{n}$ computed for 2048 data points (the whole set) to the $R_{n}$ calculated for smaller sets of data extracted from the whole range. Although the shorter data sets imply a cut-off at a slightly higher (8x) low frequency limit, good agreement between the overall $R_{n}$ and the $R_{n}$ of each sub-group without any significant trend shows that system is in steady state and noise resistance does not change significantly over time. $R_{n}$ values of 6 sets of data are within $1 \times \sigma$ (standard deviation) around the mean value which shows a normal distribution of data. This method may be considered an efficient way to assess the state of corroding cell in laboratory. In practical on-site applications, several measurements may also be made and the $R_{n}$ be compared to ensure that steady state has been reached.

\section{Figure 10}

\subsection{Role of reference electrode}

A minimal contribution of a good RE in the noise signal is desirable when used for electrochemical noise measurement. The $\mathrm{Ag} / \mathrm{AgCl}$ reference is well known to be light sensitive and may generate reasonable level of noise, thus it is suggested to be avoided for electrochemical noise measurement [27]. It should be noted that, similar to the single substrate [14] and NOCS [19] arrangements, the contribution of RE in the noise current signal is as important as noise potential when the single cell arrangement is used. This is unlike the traditional salt-bridge arrangements where RE is only involved in measuring the noise potential. It has been shown in our previous work [22] that the 
electrode with higher impedance and smaller standard deviation of noise current dominates the noise current measurement. Cottis and Turgoose [28] explain that a high resistance electrode confines the current passing through the circuit, therefore the electrode with higher resistance will dominate the noise current within an asymmetric couple. In simple terms the reference electrode noise will be applied across the cell by the action of the potentiostat. This will cause a current to flow through the cell. Consequently, noise potential power of the reference electrode will generate a

current power given by $\overline{E_{n, r e f}^{2}} / Z_{s}^{2}$, where $\overline{E_{n, r e f}^{2}}$ is the noise potential power of the reference electrode (defined as variance of noise signal) and $Z_{s}$ is the impedance of the cell generating the noise current [27]. Therefore a larger impedance of the corroding sample will reduce the influence of the RE on the noise current signal.

Concerning the noise potential, as little as possible standard deviation of RE is desirable so that the measured noise potential signal is only reflected from sample perturbation. Figures 11 shows the standard deviation of noise current and potential generated by $\mathrm{SCE}, \mathrm{Ag} / \mathrm{AgCl}$ reference electrodes and the samples used for this study. The noise current generated by SCE is very close to the noise generated by a low resistance painted sample. This reveals the practical limit of the SCE used for this study and suggests that the use of SCE for measuring the electrochemical noise current of very low resistance and noisy samples should be avoided. Further studies on other low impedance REs is required to find a better RE which does not impede the noise current generated by specimen. Figure 11 also shows 7 to 10 orders of magnitude lower noise potential generated by SCE compared to painted samples. It also shows the relatively noisy characteristic of $\mathrm{Ag} / \mathrm{AgCl}$ reference electrode which explains why $\mathrm{Ag} / \mathrm{AgCl} \mathrm{REs}$ are not recommended for electrochemical noise measurement.

Figure 11

It should be noted that whereas the potential drifts over time in open circuit, using potentiostatic noise current measurements results in an extremely small superimposed DC current (typically 1-2 pA for the high-performance coating). This is a result of superimposed potential in the second step of measurement when measuring the noise current. The DC current drifts over time, but this does not appear to affect the $R_{n}$ measurement significantly if data is treated appropriately and DC trend is removed from the raw noise data. This is evidenced by the good repeatability and agreement with the results of other methods (Figure 5).

Instrumental noise was also checked by measuring the electrical noise current and potential simultaneously without any electrochemical noise source being connected. The standard deviation of the instrumental noise was measured $1.09 \mathrm{E}-11$ for the noise current and zero for the noise potential. The actual noise current and potential data are shown in Figure 12 respectively. No evidence of systematic noise was observed which indicates acceptable filtering on the noise of power source. Therefore the current noise can be generated by the instrument and/or the computer. It should be noted that the electrical noise current also indicates the limitation of instrument in measuring electrochemical noise of the extremely low currents.

Figure 12 
Use of asymmetric electrodes for measuring electrochemical noise of uncoated metal has been extensively discussed by Cottis [29], Lowe et al. [30] and Bertocci et al. [31]. It was shown that in an asymmetric couple with different resistances, the electrode with higher resistance controls the current flowing through the ZRA thus dominating the current noise signal. The second working electrode with lower resistance and larger active surface dominates the potential fluctuation. All of these experimental and theoretical works were performed on bare metal substrates where the impedances are much lower than coated substrates and the current flow is not controlled by barrier properties of a coating. Bautista and Huet [32] discussed the theoretical aspects of ENM on asymmetric coated electrodes where one electrode has higher impedance than the other. It was shown that the current is governed by the electrode with higher impedance while the other electrode controls the potential fluctuation. In the SC arrangement it is believed that high impedance of the polymer film governs the current noise (similar to the model proposed by Bautista and Huet [32] and Cottis [29]) while the non-noisy SCE does not contribute to the potential noise leading to a relatively independent noise signal mainly reflecting characteristics of the sole working electrode.

It should be remembered that the parameters compared here (all measures of $Z$ at low frequency) may correspond to the ionic resistance of the coating, but in some cases (where two distinct semicircles appear in a Nyquist plot) $R_{n}$ may represent the sum of the coating resistance and the polarization resistance of the substrate. As it happens in these particular samples no evidence of two time constants was apparent from the Bode plots. The overall conformance of the $R_{n}$ with $R_{s n}\left(f_{\min }\right)$ implies the resistive behaviour (frequency independent) of coating rather than capacitive behaviour over the frequency at which the noise resistance was measured. This may suggest that, in most cases, ions have penetrated into the paint film at the time of measurement and the system was in a steady state.

\section{Summary and Conclusion}

A new arrangement for electrochemical noise data collection has been proposed and utilized to examine the protective properties of a set of organic coatings. The reliability of the method was checked with complementary DC and EIS measurements.

The noise resistance $\left(R_{n}\right)$ calculated with single cell arrangement was in line with the impedance IZI measured by EIS at $0.01 \mathrm{~Hz}$ and with the DC resistance.

Consistency of the measured data was examined by dividing the whole range of data into several data sets. Noise resistance was measured for each group separately and compared to the overall noise resistance. Good agreement was observed between the $R_{n}$ from each segment and the overall $\mathrm{R}_{\mathrm{n}}$.

Further verification was made by transferring time domain data into the frequency domain and comparison between spectral noise resistance $\left(R_{s n}\right)$ and the Bode plot from EIS. Spectral noise showed a very good compliance with $|Z(f)|$. Also the current and potential PSD plots were compared to examine the independency of $R_{n}$ from sampling frequency. Similarity of the slopes of 
current and potential PSD plots reveals that $R_{n}$ is frequency-independent and therefore it is a parameter of the corroding system.

This new arrangement shows promise to solve the problem of measuring electrochemical noise where preparing two isolated working electrodes is difficult, or characterization of one particular area is required. Although more work should be done to further examine the limitations of the SC arrangement, it has shown great promise to be a functional method for electrochemical noise data collection on a single working electrode.

\section{Acknowledgments}

The authors wish to thank Pronto Paints Co., Liquid Plastics Ltd and Akzo-Nobel for providing material and samples and to the School of Science and Technology at the University of Northampton for the provision of laboratory facilities. Thanks are also expressed to Prof. D. Tallman, Department of Coatings and Polymeric Materials, North Dakota State University, USA, for valuable discussions and comments. Provision of equipments and lab space for complementary experiments by Prof. G.G. Wallace and Prof. S.M. Moulton at Intelligent Polymer Research Institute, University of Wollongong, Australia, is also gratefully acknowledged.

\section{References}

[1] J. Mojica, E. Garcia, F.J. Rodriguez, J. Genescá, Prog. Org. Coat. 42 (2001) 218-225.

[2] D.J. Mills, S.J. Mabbutt, G.P. Bierwagen, Prog. Org. Coat. 46 (2003) 176-181.

[3] H.A.A. Al-Mazeedi, R.A. Cottis, Electrochim. Acta. 49 (2004) 2787-2793.

[4] Y. Tan, Sens. Actuators, B-Chem. 139 (2009) 688-698.

[5] Y.F. Cheng, M. Wilmott, J.L. Luo, Appl. Surf. Sci. 152 (1999) 161-168.

[6] D. Eden, M. Hoffman, B. Skerry, in: R.A. Dickie, F.L. Floyd (Eds.), Polymeric Material for Corrosion Control, American Chemical Society, Washington DC, 1986, pp. 36-47.

[7] B. Skerry, D. Eden, Prog. Org. Coat. 15 (1987) 269-285.

[8] B.S. Skerry, A. Alavi, K.I. Lindgren, J. Coat. Technol. 765 (1988) 97-106.

[9] Q.L. Thu, G.P. Bierwagen, S. Touzain, Prog. Org. Coat. 42 (2001) 179-187.

[10] S.J. Mabbutt, D.J. Mills, C.P. Woodcock, Prog. Org. Coat. 59 (2007) 192-196.

[11] A. M. Homborg, T. Tinga, X. Zhang, E.P.M. van Westing, P.J. Oonincx, G.M. Ferrari, et al., Electrochim. Acta. 104 (2013) 84-93.

[12] S. Ritter, H.P. Seifert, Mater. Corros. 64 (2013) 683-690.

[13] H.S. Klapper, J. Goellner, A. Burkert, A. Heyn, Corros. Sci. 75 (2013) 239-247. 
[14] S.J. Mabbutt, D.J. Mills, Br. Corros. J. 33 (1998) 158-160.

[15] G.P. Bierwagen, X. Wang, D.E. Tallman, Prog. Org. Coat. 46 (2003) 163-175.

[16] D.J. Mills, S.J. Mabbutt, in: E. Kálmán (Ed.), 7th International Symposium on Electrochemical Methods in Corrosion Research, Budapest, Hungary, 2000, paper no. 145.

[17] S.J. Mabbutt, G.P. Bierwagen, D.J. Mills, Anti-Corros. Methods Mater. 49 (2002) 264-269.

[18] S.J. Mabbutt, D.J. Mills, Surf. Coat. Int. B. 84 (2001) 277.

[19] C.P. Woodcock, D.J. Mills, H.T. Singh, J. Corros. Sci. Eng. 8 (2004) 1-10.

[20] D.J. Mills, J. Corros. Sci. Eng. 8 (2004) paper 12.

[21] D.J. Mills, S.S. Jamali, M.T. Tobiszewski, Prog. Org. Coat. 74 (2012) 385-390.

[22] S.S. Jamali, D.J. Mills, C.P. Woodcock, ECS Trans. 24 (2010) 115-125.

[23] F. Mansfeld, L.T. Han, C.C. Lee, G. Zhang, Electrochim. Acta. 43 (1998) 2933-2945.

[24] M. Hernández, J. Genescá, J. Uruchurtu, A. Barba, Corros. Sci. 51 (2009) 499-510.

[25] F. Mansfeld, C. Lee, J. Electrochem. Soc. 144 (1997) 2068-2071.

[26] F. Mansfeld, C.C. Lee, G. Zhang, Electrochim. Acta. 43 (1998) 435-438.

[27] R.A. Cottis, S. Turgoose, in: B.C. Syrett (Ed.), Electrochemical Impedance and Noise, NACE International, Houston, TX, USA, 1999, p. 58.

[28] R.A. Cottis, S. Turgoose, in: B.C. Syrett (Ed.), Electrochemical Impedance and Noise, NACE International, Houston, TX, USA, 1999, p. 90.

[29] R.A. Cottis, Electrochim. Acta. 52 (2007) 7585-7589.

[30] A.M. Lowe, H. Eren, S.I. Bailey, Corros. Sci. 45 (2003) 941-955.

[31] A. Bautista, U. Bertocci, F. Huet, J. Electrochem. Soc. 148 (2001) B412.

[32] A. Bautista, F. Huet, J. Electrochem. Soc. 146 (1999) 1730-1736. 
Figure 1 Standard "Salt-Bridge" arrangement for electrochemical noise measurement.

Figure 2 Schematic laboratory set-up for ENM with Single Substrate (SS) arrangement.

Figure 3 Schematic laboratory set-up for ENM with NOCS arrangement.

Figure 4 Single cell (SC) arrangement for electrochemical noise measurement.

Figure 5 Comparison between values obtained by DC method, EIS and ENM (SC arrangement) for a range of coating resistances from low to very high.

Figure 6 Reproducibility of SC arrangement for testing different areas of a painted metal with close resistances.

Table 1 Statistical analysis of reproducibility and comparison between DC measurement, AC impedance and ENM with SC arrangement based on three measurements on the alkyd coating.

Figure 7 Noise data analysis in frequency domain for (a) cell 1, (b) cell 2 and (c) cell 3 on the alkyd paint with moderate resistance, and comparison with Bode plot (form EIS), $R_{D C}$ and $R_{n}$.

Figure 8 Noise data analysis in frequency domain using FFT and MEM methods and comparison with Bode plot (from EIS), $R_{D C}$ and $R_{n}$ for the epoxy/PU coating system.

Figure 9 PSD plots of current (left) and potential (right) data obtained by MEM for the epoxy/PU coating system.

Figure $10 R_{n}$ values calculated from a 2048 point data set and from smaller sections of 256 data point obtained by dividing the entire range data into eight equal sections.

Figure 11 Standard deviation of the noise current and noise potential generated by SCE and $\mathrm{Ag} / \mathrm{AgCl}$ reference electrode and painted samples.

Figure 12 Instrumental current and potential noise of the $\mathrm{CH}$ instruments Potentiostat/Galvanostat model 660D measured in an open circuit without electrodes. 


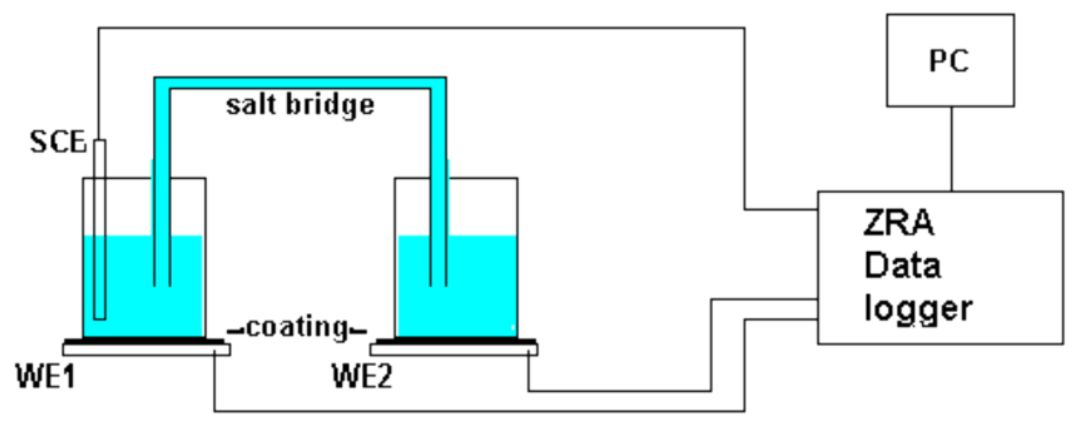

Figure 1

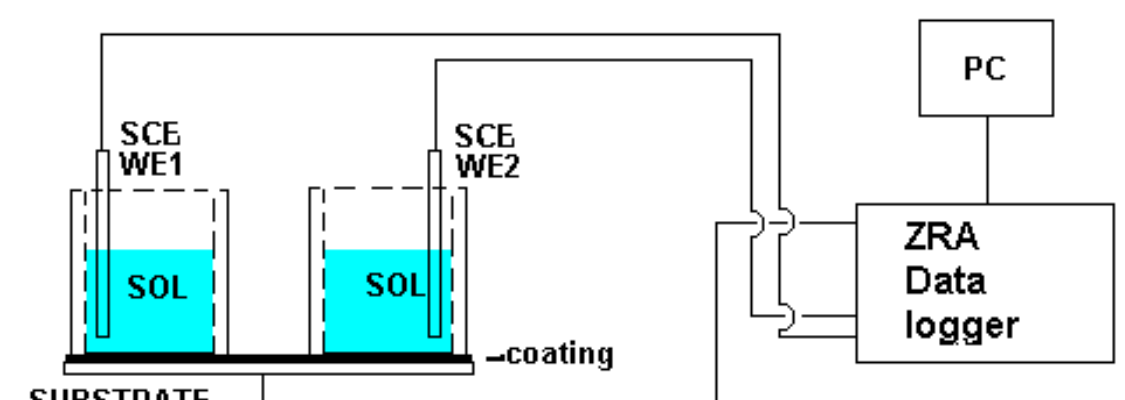

SUBSTRATE

Bridge / Ref Electrode

Figure 2

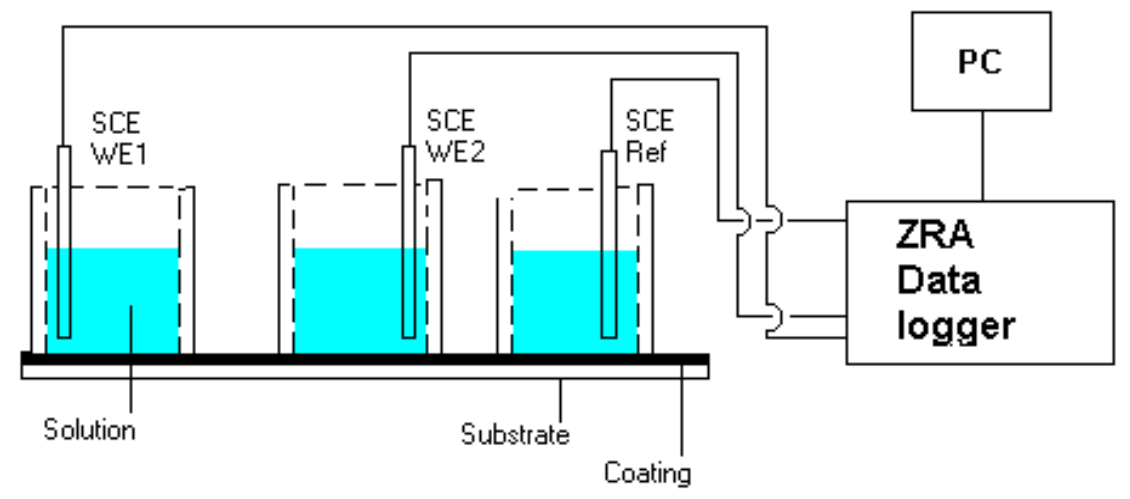

Figure 3

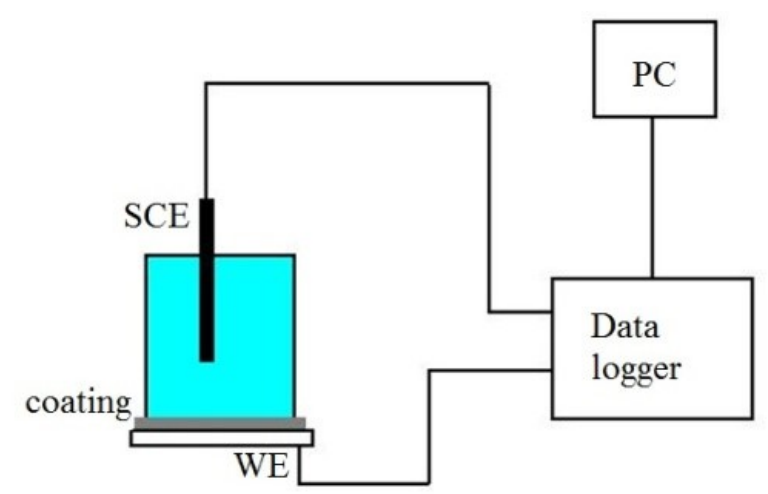

Figure 4 


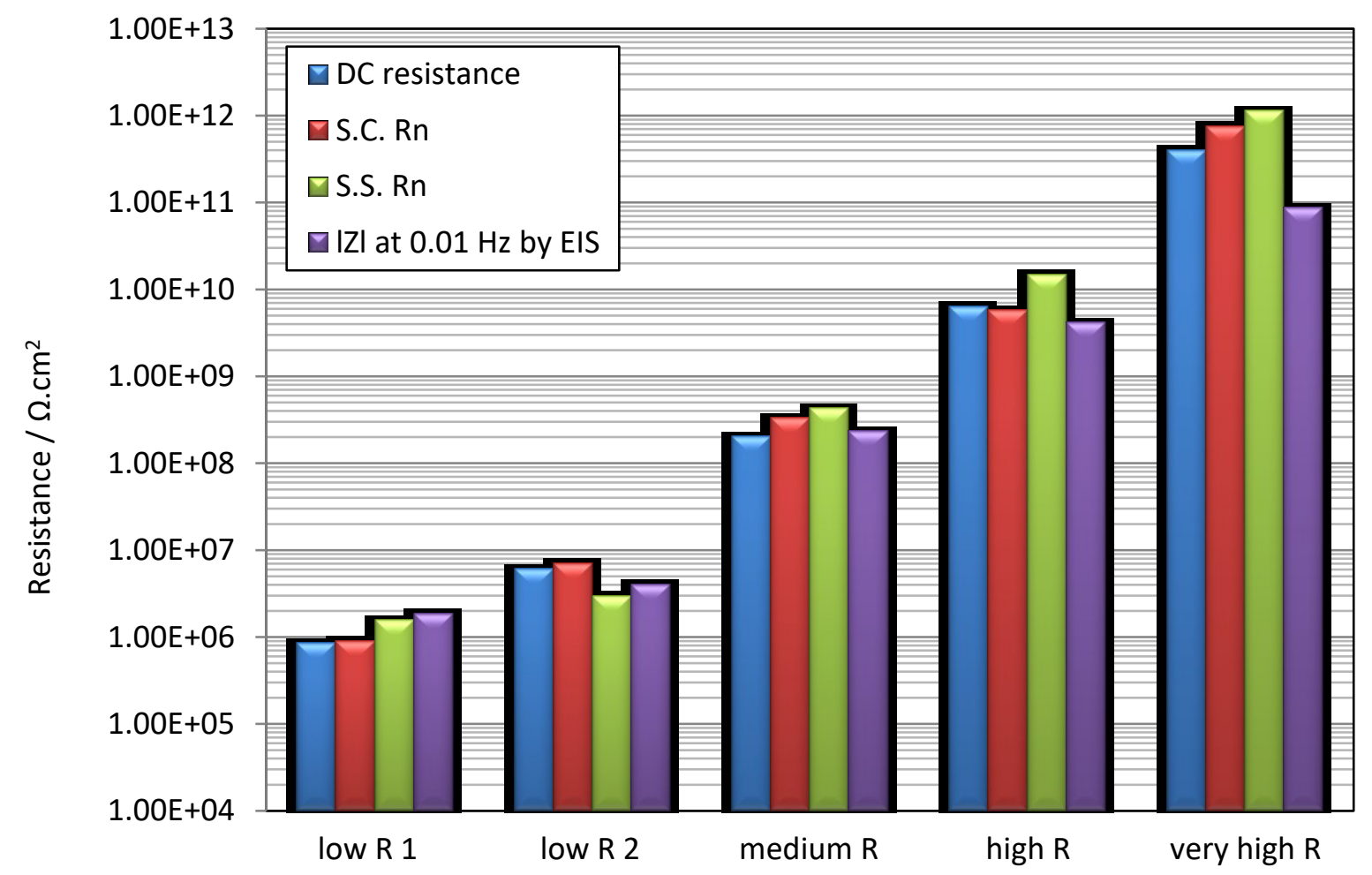

Figure 5

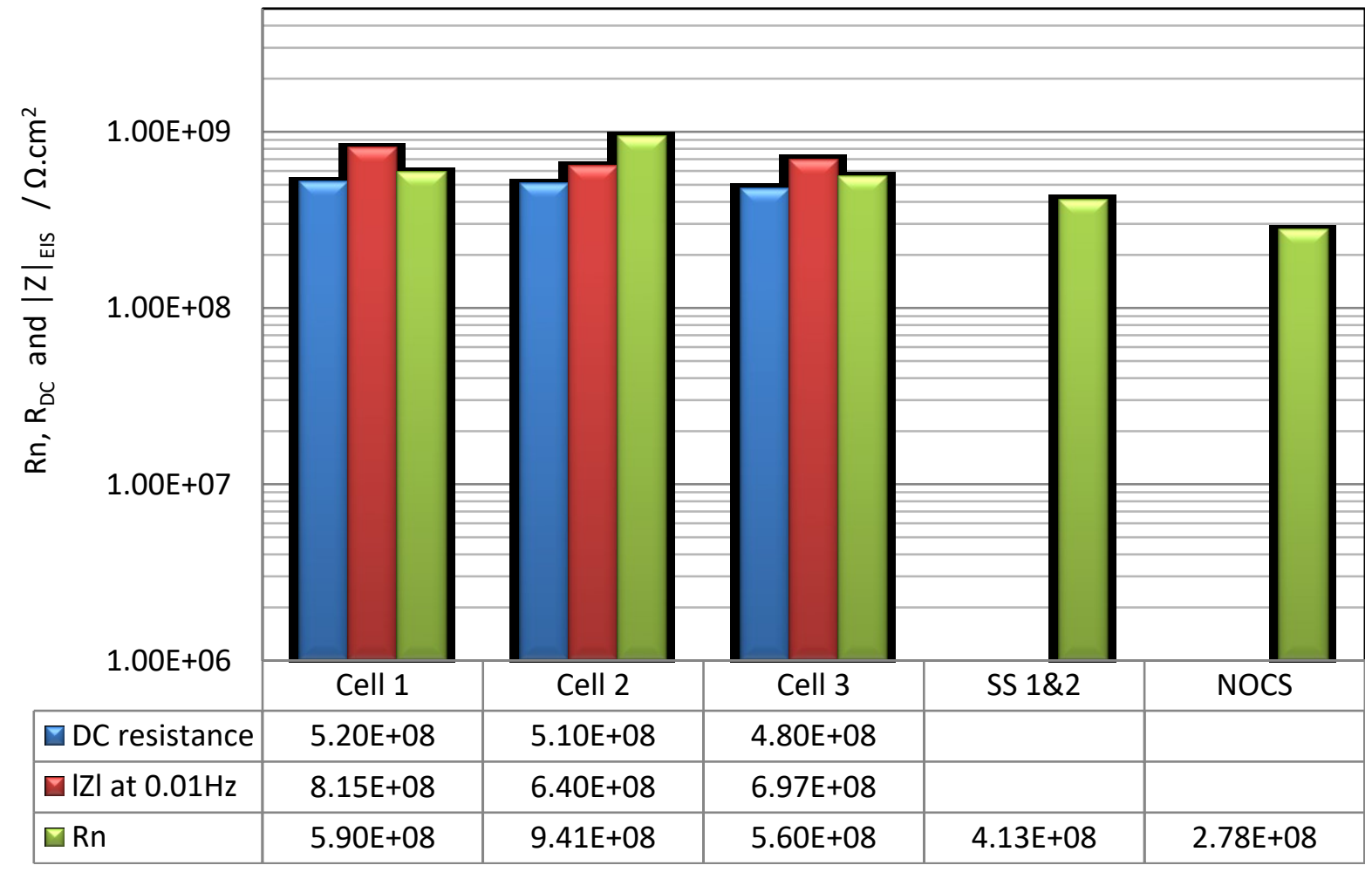

Figure 6 
Table 1

\begin{tabular}{|l|l|l|l|l|l|l|}
\hline & Cell 1 & Cell 2 & Cell 3 & ST. DeV & mean & CV \\
\hline DC resistance & $5.20 \mathrm{E}+08$ & $5.10 \mathrm{E}+08$ & $4.80 \mathrm{E}+08$ & $2.08 \mathrm{E}+07$ & $5.03 \mathrm{E}+08$ & 0.04 \\
\hline $\mathrm{IZ}$ at ${ }_{0.01 \mathrm{~Hz}}$ & $8.15 \mathrm{E}+08$ & $6.40 \mathrm{E}+08$ & $6.97 \mathrm{E}+08$ & $8.93 \mathrm{E}+07$ & $7.17 \mathrm{E}+08$ & 0.12 \\
\hline $\mathrm{R}_{\mathrm{n}}$ & $5.90 \mathrm{E}+08$ & $9.41 \mathrm{E}+08$ & $5.60 \mathrm{E}+08$ & $2.12 \mathrm{E}+08$ & $6.97 \mathrm{E}+08$ & 0.30 \\
\hline
\end{tabular}
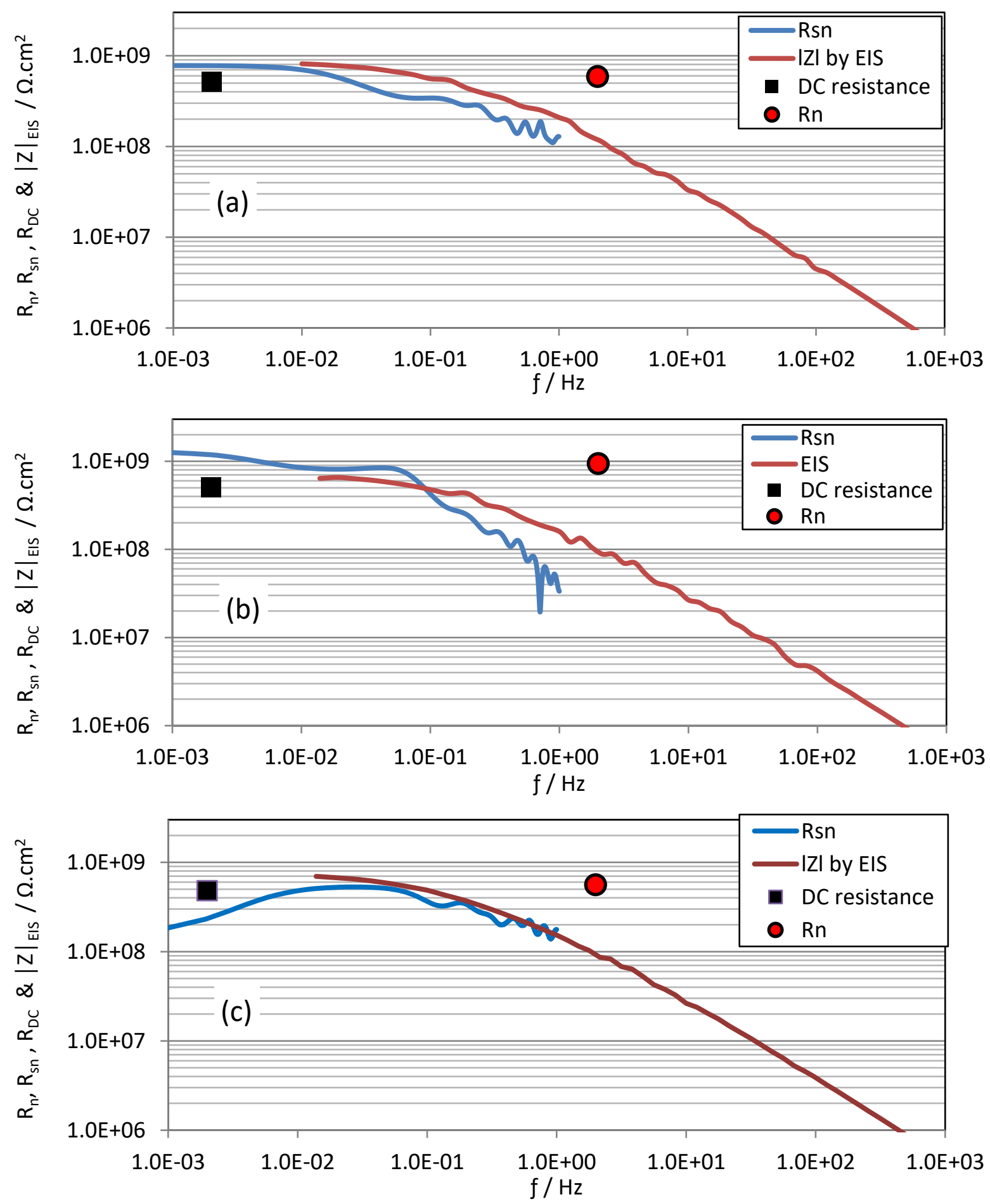

Figure 7 


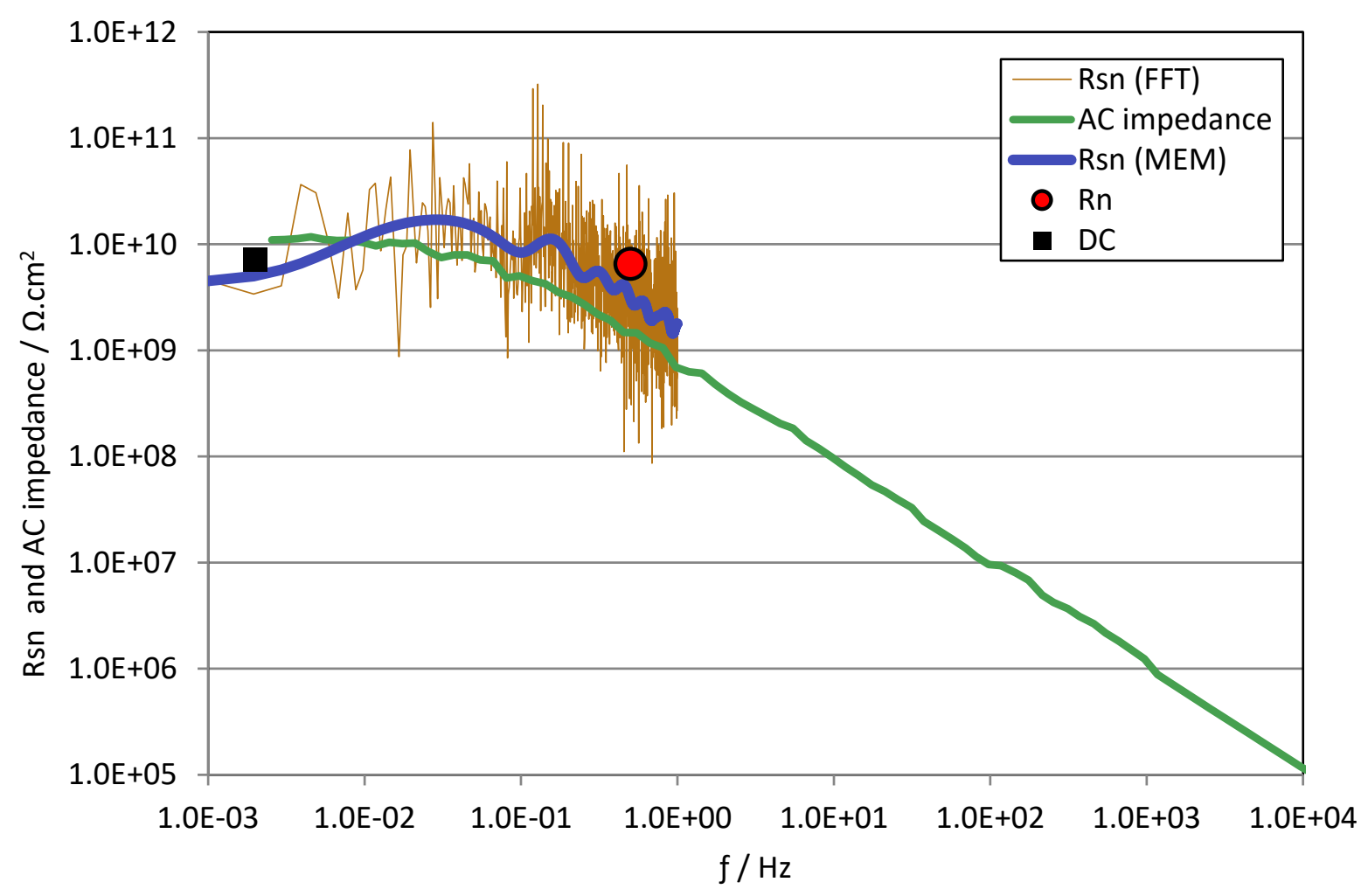

Figure 8
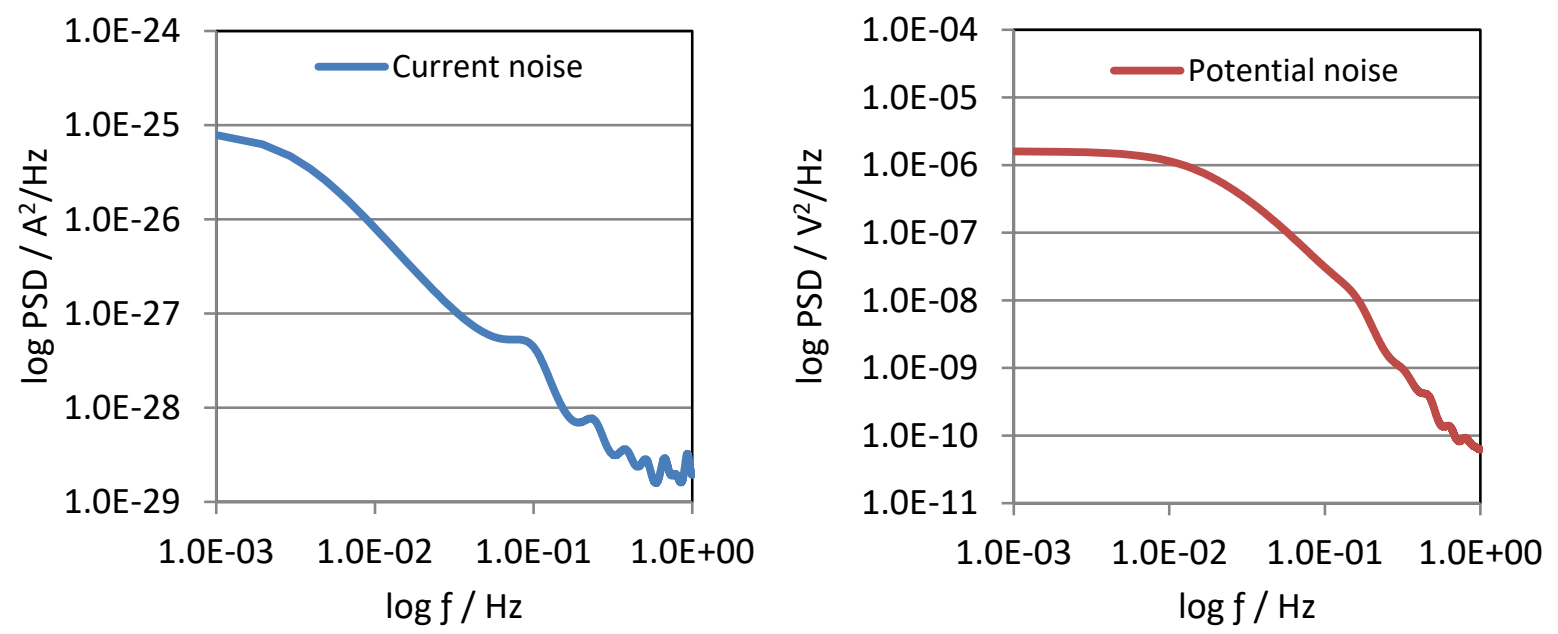

Figure 9 


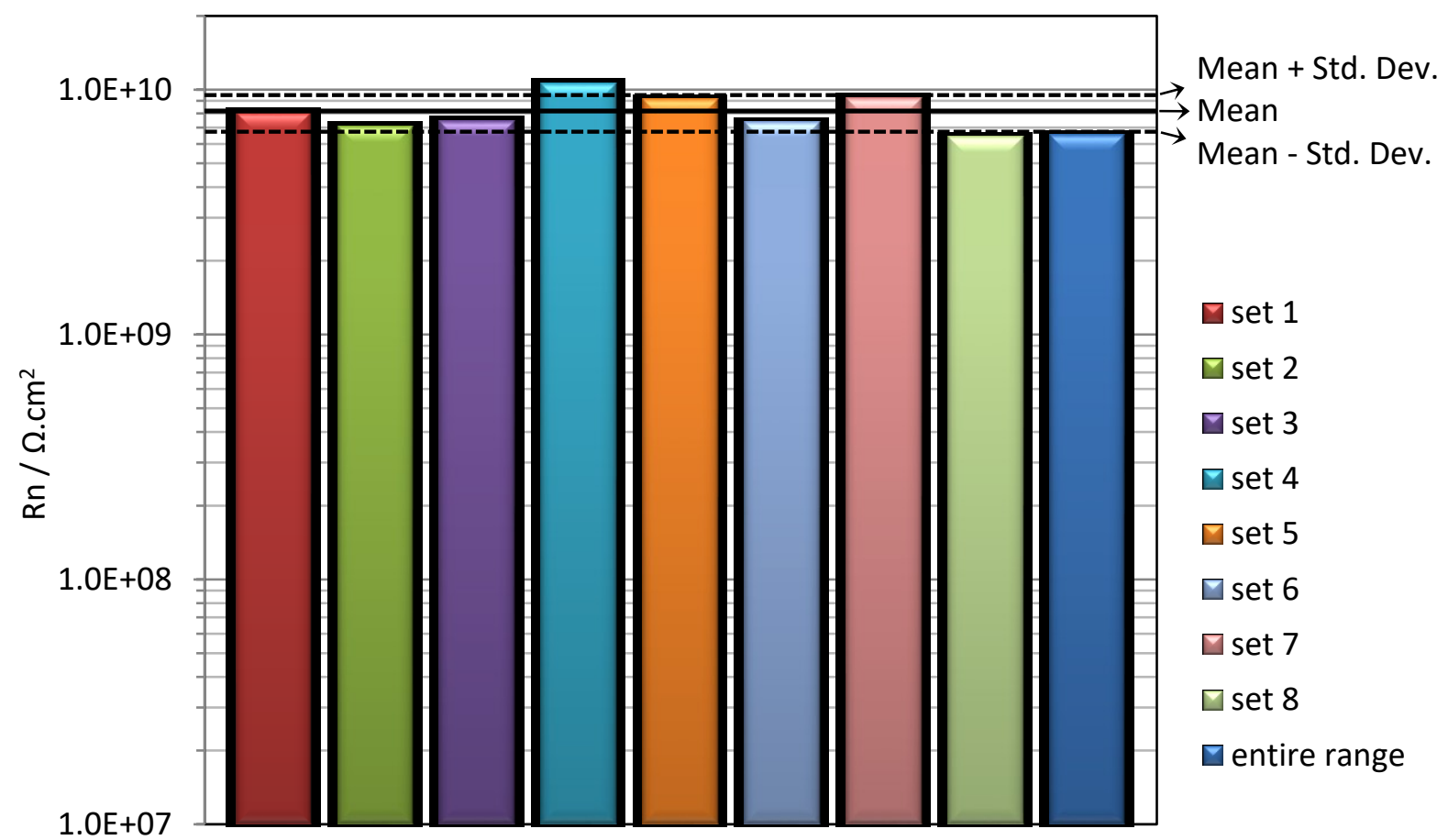

Figure 10

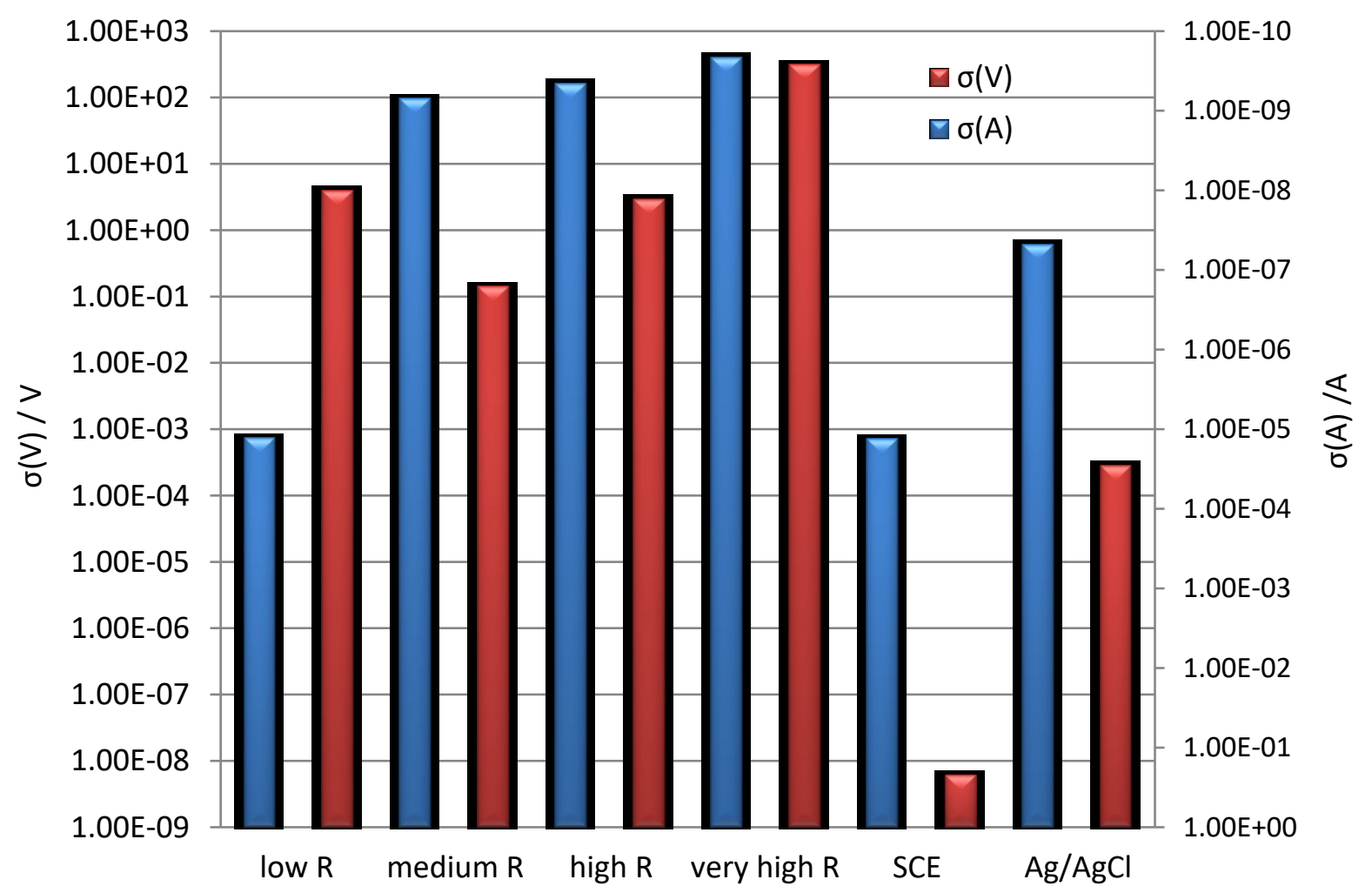

Figure 11 


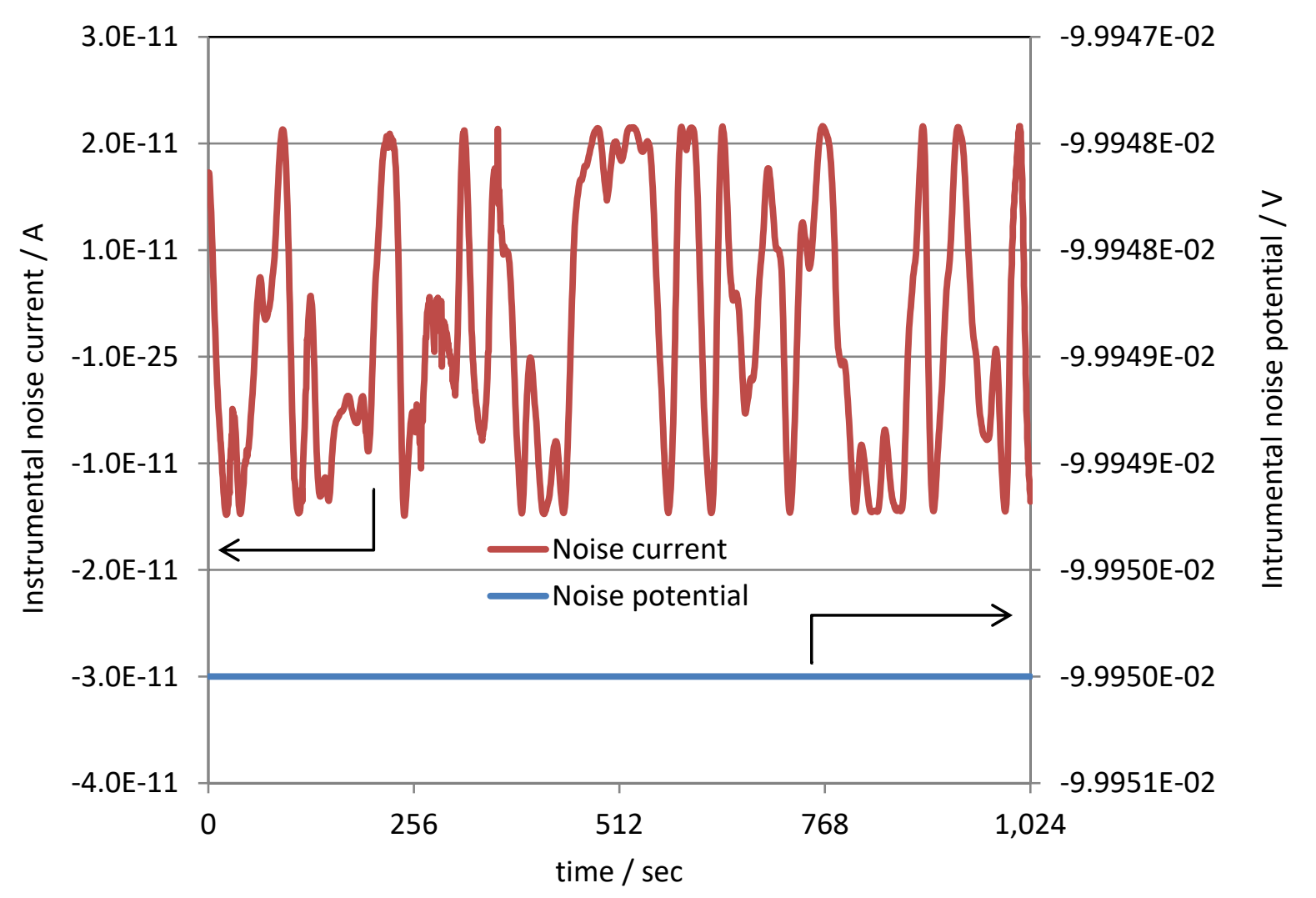

Figure 12 